\title{
Amtseid à la Obama - Verfassungsrechtliche Grundfragen und Probleme des Amtseids nach dem Grundgesetz
}

\author{
Von Rechtsassessor Walter Haensle, München*
}

Das Grundgesetz schreibt sowohl für den Bundespräsidenten als auch für den Bundeskanzler und die Bundesminister einen Amtseid als zentrales Element ihrer Amtsübernahme vor.

Dabei hat der Amtseid in Deutschland eine lange Verfassungstradition und auch in der heutigen Zeit seine Bedeutung nicht verloren. In seiner staatsrechtlichen Bedeutung stellt der Amtseid den Schnittpunkt von Staatsorganisationsrecht, Verfassungstradition und Politik dar.

Man mag beim Amtseid deswegen vielleicht sogar von den Wurzeln des Staatsrechts an sich sprechen. Es gibt wenige staatliche Akte, in denen sich das Selbstverständnis des Staates so deutlich offenbart wie beim Amtseid der höchsten staatlichen Organe. Dabei stellen sich hier Grundfragen des Staatsrechts, die folgender Beitrag erörtert.

\section{A. Einleitung}

Die Inauguration des neu gewählten US-Präsidenten Barack Obama Ende Januar 2009 wurde weltweit mit großer Freude und Anteilnahme aufgenommen. Umso größer war dann die Aufregung, als es zu einem Versprecher an der wichtigsten Stelle der Veranstaltung kam, der Vereidigung Barack Obamas auf den Stufen des Kapitols. Nachdem der perfekt durchorganisierte Tag bis dahin völlig reibungslos abgelaufen war, unterlief dem Vorsitzenden Richter des Supreme Court John Roberts ein »kleiner» Lapsus. Beim Vorsprechen vertauschte Roberts einige der drei Dutzend von der Verfassung vorgegebenen Worte für die Vereidigung. Als er zum ersten Mal den Amtseid vorsprach, versetzte er das Wort »faithfully« (»getreulich«) von der Mitte des Satzes an dessen Ende. Dadurch verwirrte er Obama, der nur einen Teil der Formel nachsprach und dann abbrach. Beim zwei-

* Der Autor ist wissenschaftlicher Mitarbeiter am Lehrstuhl für Öffentliches Wirtschafts- und Steuerrecht an der Ludwig-Maximilians-Universität München. Für Rat und Hilfe zu diesem Aufsatz danke ich besonders Herrn Professor Dr. Kersten und Herrn Professor Dr. Lehner. 
ten Mal ließ Roberts dann das Wort »execute« (»verwalten«) aus. Schließlich wiederholte Obama die erste falsche Version ${ }^{1}$. Am nächsten Tag beteuerte das US-Präsidialamt, dass der Eid trotzdem seine Gültigkeit hatte. Zur Sicherheit wurde aber die Vereidigung noch einmal im Weißen Haus mit nur kleinem Publikum nachgeholt ${ }^{2}$.

Zwar findet die Vereidigung des Bundespräsidenten und des Bundeskanzlers in Deutschland (zumindest bisher) bei weitem kein so mediales Echo wie die Amtseinführung des US-Präsidenten. Dennoch mussten sich sowohl das höchste deutsche Gericht als auch die bundesdeutsche Nachkriegsgesellschaft mit dem Beamteneid beschäftigen, der mit dem Amtseid strukturell verwandt ist.

In einer grundlegenden Entscheidung vom 17.12.1953 zu Art. 131 GG entschied das BVerfG, dass alle Beamtenverhältnisse am 8. Mai 1945 erloschen sind. Entscheidendes Argument hierfür war, dass im »Dritten Reich « alle Beamten persönlich auf den »Führer« einen Treueid leisten mussten, der dem »Beamtenverhältnis eine neue, den bisherigen Inhalt umstürzende Grundlage« gab. Durch diesen Eid hatte das Beamtenverhältnis eine Rechtsgrundlage erhalten, die vom Bestehen der nationalsozialistischen Herrschaftsform abhängig war. Mit Untergang des NSStaates erloschen die Beamtenverhältnisse ipso facto ${ }^{3}$.

Im sogenannten Remer-Prozess ${ }^{4}$ vor noch nicht ganz 60 Jahren wies der spätere Generalstaatsanwalt Fritz Bauer mit Hilfe von vier Gutachten zweier evangelischer, eines katholischen Theologen und eines ehemaligen Generals nach, dass die Widerstandskämpfer des 20. Juli 1944 weder Landes- und Hochverrat noch Eidbruch begangen hatten. Da der unsägliche Eid auf Hitler unsittlich war, konnten Stauffenberg und seine Mitstreiter diesen Eid gar nicht brechen ${ }^{5}$. Erst mit diesem Prozess wurden die Widerstandskämpfer vom Makel des Eidbruchs und des Landes- und Hochverrats rehabilitiert.

Die in diesen Fällen gezeigte rechtliche und politische Bedeutung des Eides ist Anlass für folgende Untersuchung.

\section{B. Begriff, Geschichte, Funktion}

\section{Definition}

Nach der lexikalen Definition ist der Eid eine »feierliche Bekräftigung einer Aussage mit oder ohne Verbalanrufung Gottes. $\ll^{6}$ Wird er vor Übernahme bestimmter Pflichten eines Amtes gesprochen, so nennt man ihn einen Amtseid ${ }^{7}$. Das Amt ist dabei regelmäßig eine Position innerhalb des Staates oder einer Religionsgemeinschaft ${ }^{8}$.

Der Eid, den die Organwalter des Staates leisten, wird auch speziell als politischer Eid bezeichnet ${ }^{9}$. Dabei ist einerseits zwischen dem promissorischen Eid (iuramentum promissorium), bei dem der Schwörende verspricht, in Zukunft etwas zu tun oder zu unterlassen, und andererseits dem assertorischen Eid (iuramentum assertorium) zu unterscheiden. Bei letzterem wird versichert, dass eine bestimmte Aussage der Wahrheit entspricht ${ }^{10}$. Der Eid, den der Bundespräsident nach Art. 56 GG sowie der Bundeskanzler und die Bundesminister nach Art. 64 II GG zu leisten haben, ist promissorischer Natur ${ }^{11}$.

\section{Geschichte des Eides in der deutschen Verfassungs- geschichte}

Die Pflicht zur politischen Eidesleistung kann in Deutschland auf eine lange Verfassungstradition zurückblicken. Sie reicht bis in die Zeiten des Heiligen Römischen Reiches deutscher Nation zurück. So musste schon nach dem Sachsenspiegel der römischdeutsche Kaiser geloben, »dat he recht sterke unde unrecht krenke unde it rike voresta an sine rechte als he künne unde moge (»dass er das Recht stärke und das Unrecht schwäche und dem
Reich vorstehe an seinem Rechte, als er könne und vermöge « $)^{12}$. Auch die Paulskirchenverfassung von 1849 sah in $\$ 190$ I eine Pflicht des Kaisers zur Eidesleistung auf die Reichsverfassung vor. Dem folgten sowohl die Preußische Verfassungsurkunde von 1850 als auch die meisten anderen Verfassungen der deutschen Teilstaaten des 19. Jahrhunderts.

Dies war in der Ära der Reaktion aber keine Selbstverständlichkeit. So lehnte der preußische König Friedrich Wilhelm IV. selbst den Eid zeitlebens ab. Nur dem Insistieren einiger beherzter Politiker ist es zu verdanken, dass er trotzdem den Eid auf die revidierte Verfassung leistete, wie es Art. 112 der oktroyierten preußischen Verfassung von 1848 vorsah. Einzig die Reichsverfassung von 1871 vermied für den deutschen Kaiser eine Eidesregelung. Dennoch gaben sowohl Kaiser Friedrich III. als auch Kaiser Wilhelm II. beim Regierungsantritt ein - freilich rechtlich nicht bindendes - freiwilliges Treuegelöbnis auf die Reichsverfassung $\mathrm{ab}^{13}$. Erst die Weimarer Reichsverfassung knüpfte wieder an die deutsche Tradition an, indem sie in Art. 42 WRV dem demokratisch gewählten Reichspräsidenten einen in der Verfassung textlich genau festgelegten Eid vorgab. Dabei entsprach der Wortlaut von Art. 42 WRV im Wesentlichen dem heutigen Art. 56 GG.

Vor dem Hintergrund der negativen Erfahrungen der Weimarer Republik erweiterte der Parlamentarische Rat in Art. 56 GG die Eidesformel der Weimarer Reichsverfassung um die aktive Pflicht, das Grundgesetz zu verteidigen ${ }^{14}$. Damit hat das Konzept der Wehrhaften Demokratie des Grundgesetzes seinen Niederschlag auch in der Eidesformel gefunden.

Dieser gegenüber der Weimarer Reichsverfassung verstärkte Eid des Bundespräsidenten wurde vom Parlamentarischen Rat

1 Die Eidesformel der US-Verfassung lautet korrekt: »I do solemnly swear (or affirm) that I will faithfully execute the Office of President of the United States, and will to the best of my ability, preserve, protect and defend the Constitution of the United States."

2 Süddeutsche Zeitung Nr. 16 vom 21.01.09, S 1, 3, 5; SZ Nr. 18 vom 23.01.09, S 5 .

3 BVerfGE 3, 58, 98ff, $114 \mathrm{f}$; vgl auch die kritische Besprechung bei KerN DVBl 1954, 273.

4 Der Remer-Prozess wegen übler Nachrede und Verunglimpfung des Andenkens Verstorbener fand im März 1952 vor der großen Strafkammer in Braunschweig gegen den ehemaligen Kommandeur des Wachbataillons „Großdeutschland « und späteren Rechtsextremisten Ernst Otto Remer, der an der Verhinderung des Umsturzversuches der Widerstandskämpfer des 20. Juli 1944 beteiligt war, statt. Remer hatte die Teilnehmer des 20. Juli als Landesverräter verunglimpft. Die Strafkammer verurteilte ihn deswegen zu einer Haftstrafe von drei Monaten.

5 Süddeutsche Zeitung Nr. 44 vom 23. Februar 2009, S 14.

6 Der Brockhaus in 3 Bänden, Bd I, 2. Auflage, 1995, S 441.

7 Grimmsche Deutsches Wörterbuch, Bd II, 1998, Col. 689; Der Brockhaus in 3 Bänden, Bd I, 2. Aufl, S 441.

8 So leisten bspw auch katholische Bischöfe einen Treueid auf die BRD und das jeweilige Bundesland nach Art. 16 des Konkordates von 1933. Außerdem müssen sie nach c. 380 Codex Iuris Canonici 1983 vor Amtsantritt einen Treueid ablegen, in dem sie ua auf den katholischen Glauben, die kirchliche Disziplin und zum Gehorsam gegenüber der kirchlichen Autorität verpflichtet werden.

9 Görres-Gesellschaft/Frisenhahn, Staatslexikon, Bd II, 7. Auflage, 1986, Col 157.

10 Schmidt-Bleibtreu/Klein/Butzer Kommentar zum GG, 11. Auf 2008 Art. 56 Rdn. 3; Stern Das Staatsrecht der Bundesrepublik Deutschland, Bd II, 1980, § 30 II 5 b); Sachs/Nierhaus GG Kommentar, 5. Aufl, 2009, Art. 56 Rdn. 2; v. Mangoldt/Klein/Starck/FIN K Kommentar zum Grundgesetz, Bd II, 5. Auflage, 2005, Art. 56 Rdn. 3.

11 Schmidt-Bleibtreu/Klein/Butzer (Fn. 10) Art.56 Rdn.3; SterN (Fn. 10) $\$ 30$ II 5; Sachs/Nierhaus (Fn. 10) Art. 56 Rdn. 2; v. Mangoldt/ Klein/Starck/Fink (Fn. 10) Art. 56 Rdn. 3.

12 Schmidt-Bleibtreu/Klein/Butzer (Fn. 10) Art. 56 Rdn. 1; Dreier/PerNICE Grundgesetz Kommentar, Bd II, 2. Auflage, 2006, Art. 56 Rdn. 2.

13 Huber Deutsche Verfassungsgeschichte Bd III, 3. Auflage 1988, S 52 f, $162 \mathrm{ff}, 1013$.

14 So hatte der Reichspräsident gemäß Art. 42 WRV nur »...die Verfassung und die Gesetze des Reiches zu wahren...«. 
über die Rückverweisung des Art. 64 II GG auch dem Bundeskanzler und den Bundesministern auferlegt ${ }^{15}$.

\section{Sinn und Funktion des Eides}

Die Ursprünge des Eides liegen im Christentum. Danach brachte die Eidesleistung die Bindung des Amtsträgers an die göttliche Ordnung zum Ausdruck. Zwar wird im heutigen demokratischen Staat des Grundgesetzes der Eid nicht mehr gegenüber Gott abgelegt, sondern gegenüber dem Volk als Souverän. Dennoch steht auch der Eid des Art. 56 GG (und Art. 64 II GG) in der christlichen Tradition, indem er die religiöse Beteuerung zum Regelbestand macht ${ }^{16}$.

Durch diese zusätzliche Berufung auf Gott erhält der Eid noch ein weiteres transzendentales Element, welches das Versprechen des Schwörenden verstärkt. Traditionell steht der Bruch eines solchen promissorischen Eides einem Meineid gleich und ist nach christlichem Verständnis der Verlust des Seelenheils die Folge des Eidbruchs.

Natürlich erlaubt das Grundgesetz als Ausdruck der weltanschaulichen und religiösen Neutralität des Staates sowie der Religionsfreiheit, die religiöse Beteuerung wegzulassen.

Aber auch in einem solchen Fall eines rein weltlichen Gelöbnisses ist verfassungspolitischer Zweck der Eidespflicht die feierliche ethisch-moralische Selbstbindung des Bundespräsidenten, Bundeskanzlers und Bundesministers ${ }^{17}$.

Durch den Eid wird bei seiner Abnahme die positive Einstellung des zur Eidesleistung verpflichteten Amtsträgers zur grundgesetzlichen Staatsordnung geprüft. Später soll er aufgrund seiner besonderen ethischen Bedeutung die Gewissensbedenken und die Furcht vor gesellschaftlichen Sanktionen beim Eidbruch verstärken, womit eine letzte innere Hemmschwelle errichtet wird $^{18}$. Damit soll der Eid ein Mittel zum Schutz der Verfassung und der auf ihr beruhenden Rechtsordnung $\operatorname{sein}^{19}$.

Ein weiterer Zweck ist die dem Eid zugesprochene Symbolfunktion. Diese besteht darin, die nicht unmittelbar vom Volk gewählten Amtsträger vor dem Parlament (und im Falle des Bundespräsidenten auch vor dem Bundesrat) dem Wohl des Volkes zu verpflichten ${ }^{20}$.

Schließlich sei als letzte Funktion des Eides noch die Gelegenheit des Staates zur Selbstdarstellung genannt. Die öffentliche und feierliche Vereidigung der höchsten staatlichen Funktionsträger zählt zu den wenigen integrativen Feierlichkeiten, die es in der Bundesrepublik Deutschland gibt ${ }^{21}$.

\section{Verfassungsrechtliche Grundfragen}

\section{Vereidigung des Bundespräsidenten}

Im V. Abschnitt des Grundgesetzes über den Bundespräsidenten befindet sich die zentrale verfassungsrechtliche Norm des Amtseides (Art. 56 GG), auf die alle übrigen Eidesregelungen des Grundgesetzes Bezug nehmen.

\section{Eidesverweigerung}

a) Fall 1: Der Bundespräsident ist mit den Stimmen der Mehrheit der Mitglieder der Bundesversammlung gewählt worden. Er hat auch die Wahl gegenüber dem Präsidenten des Bundestages angenommen. Die Eidesleistung verweigert der Bundespräsident beharrlich.

Nach Art. 56 GG hat der Bundespräsident den dort niedergelegten Eid mündlich vor den versammelten Mitgliedern von Bundestag und Bundesrat zu leisten. Dieser Fall, dass der von der Bundesversammlung gewählte neue Bundespräsident sich kategorisch weigert, diesen Eid zu leisten, ist bisher in der Geschichte der Bundesrepublik nicht vorgekommen ${ }^{22}$. Dennoch handelt es sich hierbei um eine Grundfrage des Staatsrechts.
Zunächst stellt sich das Problem, ob der Bundespräsident wirksam sein Amt angetreten hat. Die Beantwortung dieser Frage hängt davon ab, ob man den Amtseid des Art.56 GG eine konstitutive Wirkung beimisst. Die Kommentarliteratur ist diesbezüglich nahezu einhellig der Auffassung, dass der Amtseid des Grundgesetzes keine konstitutive Wirkung hat. Amtsantritt und Eid stünden in keinem Bedingungszusammenhang ${ }^{23}$. Mit dem Hinweis, dass dies »allgemeine Ansicht ${ }^{24}$ sei, unterbleibt jedoch regelmäßig die Untermauerung mit Argumenten.

Als erstes ist festzustellen, dass das Grundgesetz in Art. 56 GG nur vorschreibt, dass der Eid »bei seinem Amtsantritt« zu leisten ist. Nach dem Wortlaut des Art. 56 GG hängt damit der Beginn der Amtszeit nicht von der Eidesleistung ab. Vielmehr ist Art. 56 GG nur zu entnehmen, dass der Eid in unmittelbarem zeitlichem engen Zusammenhang mit dem Amtsantritt stattfinden soll ${ }^{25}$. Damit erschöpft sich diese Norm nur darin, dass sie vorschreibt, wann der Eid zu leisten ist, nicht aber, dass das Amt vom Eid abhängt. Über den Zeitpunkt des Amtsantritts selbst schweigt das Grundgesetz hingegen. Es findet sich nur eine einfachgesetzliche Regelung: Nach $\$ 10$ des Gesetzes über die Wahl des Bundespräsidenten durch die Bundesversammlung (BPräsWahlG) beginnt das Amt des Bundespräsidenten mit dem Ablauf der Amtszeit seines Vorgängers, jedoch nicht vor Eingang der Annahmeerklärung beim Präsidenten des Bundestages. Auch nach dieser Norm ist also die Eidesleistung nicht konstitutiv für den Beginn des Amtes. Weiter besteht Einigkeit darüber, dass der Eid keinerlei über die Verfassungsbestimmungen hinausgehenden Zuständigkeiten und Befugnisse des Staatsoberhauptes begrün$\operatorname{det}^{26}$. Denn er bekräftigt nur diejenigen, die sich bereits aus Verfassung und Gesetz ergeben ${ }^{27}$. Mit anderen Worten: Der Eid ist nicht kompetenzbegründender, sondern nur kompetenzausfüllender Natur ${ }^{28}$. Dies ergibt sich bereits daraus, dass der Wortlaut der Eide des Bundespräsidenten (Art.56 GG) und

15 Schmidt-Bleibtreu/Klein/Butzer (Fn. 10) Art. 56 Rdn. 1ff; Dreier/PerNiCE (Fn. 12) Art. 56 Rdn. 3 f; Dolzer/Waldhoff/Graßhof/Fritz Bonner Kommentar, 137. Lfg, Stand Dezember 2008, Art. 56 Rdn. 1.

16 Dolzer/Waldhoff/Graßhof/Fritz (Fn. 15) Art. 56 Rdn. 2; Schmidt-Bleibtreu/Klein/Butzer (Fn. 10) Art. 56 Rdn. 4; Isensee/Kirchhof/NettesHEIM Handbuch des Staatsrechts, Bd III, 3. Auflage, 2005, \$61 Rdn. 52.

17 Schmidt-Bleibtreu/Klein/Butzer (Fn. 10) Art. 56 Rdn. 4, 6; Dreier/PerNiCe (Fn. 12) Art. 56 Rdn. 9; Maunz/Dürig/Herzog Grundgesetz Kommentar, Bd IV, 53. Lfg, Stand Oktober 2008, Art. 64 Rdn. 10.

18 STERN (Fn. 10) $\$ 30$ II 5 a); v. Mangoldt/Klein/Starck/Schröder (Fn. 10) Art. 64 Rdn. 35; Schmidt-Bleibtreu/Klein/Uhle (Fn. 10) Art. 64 Rdn. 3.

19 Schmidt-Bleibtreu/Klein/Uhle (Fn. 10) Art. 64 Rdn. 28; v. Mangoldt/ Klein/Starck/Schröder (Fn. 10) Art. 64 Rdn. 36.

20 Schmidt-Bleibtreu/Klein/Butzer (Fn. 10) Art.56 Rdn.6; SchmidtBleibtreu/Klein/Uhle (Fn. 10) Art. 64 Rdn. 28; Dreier/Hermes (Fn. 12) Art. 64 Rdn. 32.

21 Schmidt-Bleibtreu/Klein/Butzer (Fn. 10) Art. 56 Rdn. 6; Maunz/Dürig/ Herzog (Fn. 17) Art. 56 Rdn. 1 Art. 64 Rdn. 32.

22 Bisher haben alle Bundespräsidenten den Eid ohne weiteres mit der religiösen Beteuerungsformel geleistet.

23 Für viele: Maunz/Dürig/Herzog (Fn. 17) Art.56 Rdn. 4f, 14, 20; v. Münch/Kunig/Hemmerich GG - Kommentar, Bd II, 5. Aufl, 2001, Art. 56 Rdn. 2; Denninger/Hoffmann-Riem/Schneider/Stein/Jekewitz Kommentar zum Grundgesetz für die BRD, Reihe Alternativkommentare, 3. Aufl, 2. Lfg August 2002, Art. 56 Rdn. 2.

$24 \mathrm{Vgl} \mathrm{nur} \mathrm{v.} \mathrm{Münch/Kunig/Hemmerich} \mathrm{(Fn.} \mathrm{23)} \mathrm{Art.} 56$ Rdn. 2; Denninger/Hoffmann-Riem/Schneider/Stein/Jekewitz (Fn. 23) Art. 56 Rdn. 2; Schmidt-Bleibtreu/Klein/Butzer (Fn. 10) Art. 56 Rdn. 7.

25 Maunz/Dürig/Herzog (Fn. 17) Art. 56 Rdn. 4.

26 Denninger/Hoffmann-Riem/Schneider/Stein/Jekewitz (Fn. 23) Art. 56 Rdn. 2; Schmidt-Bleibtreu/Klein/Butzer (Fn. 10) Art. 56 Rdn. 9; Sachs/ Nierhaus (Fn. 10) Art.56 Rdn.3; Maunz/Dürig/Herzog (Fn. 17) Art. 56 Rdn. 20; v. Münch/Kunig/Hemmerich (Fn. 23) Art. 56 Rdn. 5; v. Mangoldt/Klein/Starck/Fink (Fn. 10) Art. 56 Rdn. 5.

27 STERN (Fn. 10) \$30 II 5 c)

28 Schmidt-Bleibtreu/Klein/Butzer (Fn. 10) Art. 56 Rdn. 9; Isensee/Kirchhof/Nettesheim (Fn. 16) \$61 Rdn. 53. 
des Bundeskanzlers und -ministers (Art. 64 II GG) absolut identisch ist, obwohl deren Kompetenzen völlig anderer Art sind ${ }^{29}$. Wenn der Eid aber keine über die Einzelermächtigungen des Grundgesetzes hinausgehenden Befugnisse und Pflichten begründet, sondern nur erläutert ${ }^{30}$, so ist dies ein weiteres Argument, dass der Eid nicht konstitutiv für das Amt des Bundespräsidenten und dessen Amtsbefugnisse ist.

Ferner kann auch eine Parallele zum Diensteid der Beamten gezogen werden. So ist die Eidesleistung weder Rechtsvoraussetzung des Erwerbs der Beamteneigenschaft noch hängt die Möglichkeit der Rüge von Dienstpflichtverletzungen von seiner vorherigen Ableistung ab. Allein entscheidend ist die Ernennung. Daher ist der Diensteid nicht das konstitutive Element der Begründung des Beamtenverhältnisses ( $\$ \$ 3,7,8 \mathrm{I}$ Be$\mathrm{amtStG}^{31}$. Zwar ist der Bundespräsident ${ }^{32}$ kein Beamter, sondern steht (nach dem Rechtsgedanken des $\$ 1$ BMinG) in einem öffentlich-rechtlichen Amtsverhältnis ${ }^{33}$. Da aber dieses Amtsverhältnis Ähnlichkeiten mit dem Beamtenverhältnis aufweist ${ }^{34}$ spricht dennoch auch der Vergleich des Amtseides mit dem Diensteid für eine Verneinung der konstitutiven Wirkung des Amtseides.

Folglich kann der Bundespräsident trotz Verweigerung der Eidesleistung sein Amt wirksam antreten. Mit Annahme der Wahl erwirbt er das Amt, $\$ \$ 9$ IV, 10 BPräsWahlG ${ }^{35}$.

b) Fall 2: Aufgrund der verweigerten Eidesleistung des Bundespräsidenten überlegt sich die Staatsanwaltschaft, ob sie tätig werden muss.

Aus dem oben dargestellten Charakter des Amtseides als promissorischer Eid folgt, dass eine Verletzung des Eides bzw. eine Weigerung, ihn zu leisten, nicht als Meineid o. ä. strafbewehrt ist ${ }^{36}$. Zum einen lassen sich aus dem Text des Eides schwerlich konkrete Handlungsanweisungen oder -pflichten ableiten. Zum anderen widerspräche eine Verknüpfung von Verfassungs- und Strafrecht deutscher Rechtstradition ${ }^{37}$.

c) Fall 3: Nach Wahl und deren Annahme nimmt der Bundespräsident trotz Eidesverweigerung verschiedene Amtshandlungen (Ausfertigung von Gesetzen, Ernennung von Bundesbeamten und -richtern, Begnadigung) vor.

In Fall 3 stellt sich die Frage nach der Wirksamkeit der Amtshandlungen. In der Literatur werden hierzu verschiedene Standpunkte vertreten. Nach einer großzügigen Ansicht ist der Eid weder Rechtmäßigkeits- noch Wirksamkeitsbedingung der in amtlicher Funktion vorgenommenen Handlungen ${ }^{38}$. Eine etwaige Unterscheidung zwischen mangelndem Erlaubtsein und Gültigkeit der Amtsführung einschließlich einzelner Amtshandlungen würde keinen Sinn machen, da der Eid keine konstitutive Wirkung habe. Hat der Amtsträger sein Amt wirksam angetreten, so habe der Betreffende nicht nur die »Erlaubnis«, sondern sogar die Pflicht, alle Amtsgeschäfte in vollem Umfang wahrzunehmen. Dies folge auch daraus, dass der Eid keine weiteren Pflichten auferlege, die nicht bereits nach dem Grundgesetz geboten seien ${ }^{39}$. Danach wären alle Amtshandlungen des unvereidigten Bundespräsidenten sowohl wirksam als auch rechtmäßig.

Diese Ansicht wird aber der Tatsache nicht gerecht, dass die Verfassung die Leistung des Eides bei Amtsantritt ausdrücklich vorschreibt (Art. 56 GG). Man kann nicht ignorieren, dass die Eidesleistung eine verfassungsrechtliche Pflicht des Bundespräsidenten ist. Der Verstoß dagegen kann nicht ohne Auswirkung auf die vorgenommenen Amtshandlungen bleiben.

Außerdem verfehlt diese Ansicht das Ziel des Art. 56 GG, die Amtsausübung von Anfang an unter Eid zu stellen ${ }^{40}$. Mithin ist ihr nicht zu folgen.

Eine andere Ansicht sieht daher eine Amtshandlung vor Eidesleistung nur dann gerechtfertigt, wenn es hierfür zwingende Gründe gibt ${ }^{41}$. Dagegen ist jedoch einzuwenden, dass nicht klar ist, welche zwingenden Gründe dafür in Frage kommen. Diese Ansicht sieht sich demnach dem Verdikt der Rechtsunsicherheit ausgesetzt. Vielmehr ist auch hier anzumerken, dass die Verfassung den Eid vorschreibt. Ausnahmen oder Rechtfertigungsgründe sind nicht vorgesehen.

Die wohl herrschende Meinung hält Maßnahmen des nicht vereidigten Bundespräsidenten zwar für verfassungsrechtlich unzulässig, jedoch nicht für unwirksam ${ }^{42}$. Dem ist zuzustimmen, da dies einerseits den Stellenwert des Eides als Pflicht des Grundgesetzes berücksichtigt, andererseits nicht vor der Feststellung die Augen verschließt, dass der Eid rechtlich nicht konstitutiv für den Amtsantritt ist. Weiter drängt sich ein Vergleich mit $\$ 15$ BBG auf, wonach bei nichtiger oder zurückgenommener Ernennung eines Beamten die von diesem bereits vorgenommenen Amtshandlungen gültig bleiben. Dieser Rechtsgedanke muss erst recht dort gelten, wo die Ernennung nicht ungültig, sondern nur der Kreationsvorgang noch nicht restlos abgeschlossen ist ${ }^{43}$.

Für Fall 3 bedeutet dies, dass die vom Bundespräsidenten vorgenommenen Amtshandlungen zwar verfassungsrechtlich unzulässig, aber rechtlich wirksam sind.

d) Fall 4: Nachdem der Bundespräsident trotz allgemeiner Kritik sich vehement weigert, den Amtseid zu leisten, überlegt man sich im Bundestag, welche Sanktionsmöglichkeiten zur Verfügung stehen.

Zunächst ist die Präsidentenanklage gemäß Art.61 GG, $§ 13$ Nr. 4 BVerfGG vor dem BVerfG in Betracht zu ziehen, die zur Aberkennung des Amtes führen kann. Und in der Tat ist hier die Literatur übereinstimmend der Auffassung, dass dies die geeignetste Reaktionsmöglichkeit ist ${ }^{44}$. Antragsberechtigt sind sowohl der Bundestag als auch der Bundesrat mit jeweils einer Mehrheit von zwei Dritteln der Mitglieder bzw. Stimmen (Art. 61 I 3 GG, $\S 49$ II BVerfGG). Die Weigerung des Bundespräsidenten, den in der Verfassung vorgesehenen Eid zu leisten, wäre eine vorsätzliche Verletzung des Grundgesetzes i. S. v. Art. 61 I 1 GG, \$56 I BVerfGG. Zwar muss das BVerfG nicht zwangsläufig bei einer Verurteilung die Entfernung des Bundespräsidenten aus dem

29 Dolzer/Waldhoff/Graßhof/Fritz (Fn. 15) Art. 56 Rdn. 7.

30 F. MÜNCH Die Bundesregierung 1954 S 168.

31 Kern DVBl 1954, 273, 276.

32 Gleiches gilt für den Bundeskanzler und die Bundesminister vgl $\$ 1$ BMinG.

33 Schmidt-Bleibtreu/Klein/Butzer (Fn. 10) Art. 54 Rdn. 99: Da das Statusverhältnis des Bundespräsidenten abgesehen von der Inkompatibilitätsregelung des Art. 54 GG und dem Immunitätsschutz nach Art. 60 IV GG i. V. m. Art. 46 II-IV GG weder verfassungsrechtlich noch einfachgesetzlich geregelt ist, wird der Rechtsgedanke des $₫ 1$ BMinG herangezogen.

34 Siehe hierzu Schmidt-Bleibtreu/Klein/Uhle (Fn. 10) Art. 64 Rdn. 19.

35 So auch Schmidt-Bleibtreu/Klein/Butzer (Fn. 10) Art. 56 Rdn. 8.

36 Schmidt-Bleibtreu/Klein/Butzer (Fn. 10) Art. 56 Rdn. 3, v. Mangoldt/ Klein/Starck/Fink (Fn. 10) Art. 56 Rdn. 3

37 Maunz/Dürig/Herzog (Fn. 17) Art. 56 Rdn. 4.

38 Zur gleichgelagerten Problematik beim Eid des Bundeskanzlers, -ministers: Dreier/Hermes (Fn. 12) Art. 64 Rdn. 31; wohl auch Dolzer/Waldhoff/Graßhof/Fritz (Fn. 15) Art. 56 Rdn.3, 9; Friauf/Höfling/Busse Berliner Kommentar zum GG, Bd III, 25. Lfg, Dezember 2008, Art. 64 Rdn. 27.

39 Dolzer/Waldhoff/Graßhof/Fritz (Fn. 15) Art. 56 Rdn. 3, 9: zur gleichgelagerten Problematik des Amtseides der BMin: v. Münch/Kunig/MeyN (Fn. 23) Art. 64 Rdn. 16; Friauf/Höfling/Busse (Fn. 38) Art. 64 Rdn. 27.

40 Zum Eid des Bundeskanzlers, -ministers: und v. Mangoldt/Klein/Starck/ SChröder (Fn. 10) Art. 64 Rdn. 39.

41 v. Mangoldt/Klein/Starck/FInK (Fn. 10) Art. 56 Rdn. 4; v. Münch/Kunig/ Hemmerich (Fn. 23) Art. 56 Rdn.2; wohl auch Schmidt-Bleibtreu/ Klein/Butzer (Fn. 10) Art. 56 Rdn. 8.

42 Für viele: Stern (Fn. 10) $\$ 30$ II 5b); Maunz/Dürig/Herzog (Fn. 17) Art. 56 Rdn. 4 f und zum Eid des Bundeskanzlers, -ministers: Maunz/Dürig/Herzog (Fn. 17) Art. 64 Rdn. 37.

43 Maunz/Dürig/Herzog (Fn. 17) Art. 64 Rdn. 37.

44 Für viele: Maunz/Dürig/Herzog (Fn. 17) Art. 56 Rdn. 13; SchmidtBleibtreu/Klein/Butzer (Fn. 10) Art. 56 Rdn. 23. 
Amt anordnen, wie aus dem Wort »kann« in Art. 61 II 1 GG, $\$ 56$ II 1 BVerfGG folgt. Dennoch handelt es sich bei der Eidesverweigerung nicht um eine bloße »Lappalie« bzw. »Formalie«, sondern um eine schwerwiegende Verfassungsverletzung, die deutlich macht, dass der Bundespräsident nicht gewillt ist, seine eindeutige Verfassungspflicht zu erfüllen, so dass regelmäßig die Aberkennung des Amtes angemessen erscheint ${ }^{45}$.

Hingegen kann die bloße Verweigerung des Eides ohne weitere Erklärungen des Bundespräsidenten nicht als Amtsverzicht gewertet werden, da wie oben gezeigt der Amtseid keine konstitutive Wirkung besitzt ${ }^{46}$.

Eine Abwahl des Bundespräsidenten etwa durch die Bundesversammlung kommt nach dem Grundgesetz nicht in Betracht, da diese Möglichkeit im Grundgesetz nicht vorgesehen ist ${ }^{47}$.

Damit bleibt als weitere Sanktionsmöglichkeit nur die Organklage nach Art. 93 I Nr. 1 GG, $\$ 13$ Nr. 5 BVerfGG. Grundsätzlich ist dies ein gangbarer Weg, da hier Streit über die Pflicht des Bundespräsidenten als ein im Grundgesetz mit eigenen Rechten ausgestattetes oberstes Bundesorgan herrscht. Indessen wäre ein Organstreit angesichts einer vehementen Eidesverweigerung nicht sinnvoll und zielführend, da durch den rein feststellenden Tenor der Bundesverfassungsgerichtsentscheidung ( $\$ 67$ BVerfGG) nur die ohnehin selbstverständliche Pflicht aus Art. 56 GG klargestellt werden würde ${ }^{48}$.

\section{Abweichung beim Sprechen der Eidesformel:}

a) Fall 5: Nach Wahl und Annahme spricht der Bundespräsident vor den versammelten Mitgliedern des Bundestages und Bundesrates den Eid, lässt aber - aus Versehen - den Teil der Eidesformel »und verteidigen " weg.

Dieser dargestellte Sachverhalt dürfte dem eingangs geschilderten Fall des US-Präsidenten Obama am nächsten kommen: Unabsichtlich wird von der in der Verfassung vorgegebenen Eidesformel abgewichen.

Im vorliegenden Fall, bei dem ein Teil des Textes weggelassen wird, herrscht in der Literatur Einigkeit, dass eine solche Veränderung nicht gestattet ist ${ }^{49}$. Hierfür spricht bereits die historische Auslegung. So sah noch Art. 78 des Herrenchiemseer Entwurfs vor, auf eine Eidesformel zu verzichten. Die Eidesleistung sollte zwar auf das Grundgesetz erfolgen, in ihrem Wortlaut jedoch frei sein. Der Parlamentarische Rat hat indessen davon Abstand genommen und ist zu einer textlichen Vorgabe im Grundgesetz zurückgekehrt ${ }^{50}$. Damit entsprach er der deutschen Verfassungstradition, die Eidesformel konstitutionell vorzugeben ${ }^{51}$.

Weiter ergibt auch der Wortlaut von Art.56 GG, dass die Eidesformel unverrückbar ist. Art. 56 S. 1 GG gibt den Text genau vor. Aus Satz 2 folgt, dass die religiöse Beteuerung am Schluss der Eidesformel nicht gesprochen werden muss. Im Umkehrschluss ist daher zu folgern, dass der Rest des Eidestextes zwingend ist.

Wenn also der Text zwingend ist, begeht der Bundespräsident wenn auch nur fahrlässig - eine Verletzung der Verfassung, indem er einen Teil des Eides weglässt. Die Abänderung der Formel ist dabei der Eidesverweigerung gleichzustellen ${ }^{52}$. Was die Rechtsfolgen betrifft, ist nach oben Ausgeführtem (C I 1 a)) klar, dass der Bundespräsident aber dennoch wirksam sein Amt angetreten hat, da der Eid nicht konstitutiv ist. Trotzdem vorgenommene Amtshandlungen sind zwar wirksam, aber unzulässig ${ }^{53}$.

$\mathrm{Da}$ aber objektiv eine verfassungsrechtliche Pflicht aus Art. 56 GG nicht erfüllt ist, muss der Bundespräsident zum schnellstmöglichen Zeitpunkt die korrekte Eidesleistung wiederholen. Dies gilt insbesondere, wenn der ausgelassene Part der Formel wie im Beispielsfall elementar ist. Gerade der Passus zur aktiven Verfassungsschutzverpflichtung wurde extra in den Eid aufgenommen, um das Konzept der wehrhaften Demokratie zu bekräftigen $^{54}$.

Ohne hier auf die Vorgaben der US-Verfassung näher einzuge- hen, sei auch erwähnt, dass der amerikanische Präsident Obama ebenfalls dieses Verfahren wählte und am nächsten Tag nach der fehlerhaften Vereidigung »zur Sicherheit« den Eid wiederholte.

Regelmäßig dürfte das Wiederholen des Eides unproblematisch erfolgen, da jeder Bundespräsident seinen fahrlässigen Lapsus möglichst schnell beseitigen will. Nur wenn Streit über die Pflicht zur Wiederholung bestehen sollte, könnte im vorliegenden Fall der Weg einer Organklage nach Art. 93 I Nr. 1 GG, $\$ 13$ Nr. 5 BVerfGG beschritten werden ${ }^{55}$. Eine Präsidialanklage nach Art. 61 GG dürfte indessen ausscheiden, da der Bundespräsident das Grundgesetz nicht vorsätzlich verletzt hat. Nur wenn der Bundespräsident sich vehement weigert, den korrekten Eid zu wiederholen, ist an ein Amtsenthebungsverfahren zu denken ${ }^{56}$.

b) Fall 6: Nach Wahl und Annahme spricht der Bundespräsident vor den versammelten Mitgliedern des Bundestages und Bundesrates den Eid, beginnt aber aus religiösen Gründen nicht mit »Ich schwöre«, sondern mit "Ich gelobe«.

Dieser Beispielsfall beschäftigt sich mit dem einleitenden Satzteil »Ich schwöre«, der vom Bundespräsidenten aus religiösen Gründen ausgelassen bzw. abgewandelt wird. Tatsächlich befreit dieser Passus - trotz der Möglichkeit des Art. 56 Satz 2 GG - den Eid nicht vollständig von religiösen Implikationen. Außerdem wird von einigen religiösen Gruppen wie zum Beispiel den Mennoniten, den Herrnhutern, den Quäkern und den Zeugen Jehovas das Schwören ganz generell abgelehnt ${ }^{57}$. Aus diesem Grund steht ein Teil der Literatur diesen Worten vor dem Hintergrund der Glaubensfreiheit aus Art. 4 I GG, Art. 140 GG i. V. m. Art. 136 IV WRV kritisch gegenüber ${ }^{58}$. Daher, so diese Ansicht, muss dem Schwörenden auch eine andere Beteuerungsformel gestattet $\operatorname{sein}^{59}$.

Diese Ansicht überzeugt aber angesichts des klaren Wortlauts nicht. Auch hat das BVerfG den Eideszwang der Art. 56, Art. 64 II GG als verfassungsmäßig bestätigt. Zwar hat es anerkannt, dass auch der Eid in seiner nichtreligiösen Form durchaus die Glaubensfreiheit betrifft und deswegen der Zeugeneid aus Glaubens-

45 So auch Maunz/Dürig/Herzog (Fn. 17) Art. 56 Rdn. 13; v. Mangoldt/ Klein/Starck/Fink (Fn. 10) Art. 56 Rdn. 8; Isensee/Kirchhof/NettesHEIM (Fn. 16) \$61 Rdn. 55.

46 v. Münch/Kunig/Hemmerich (Fn. 23) Art. 56 Rdn. 2; Schmidt-Bleibtreu/Klein/Butzer (Fn. 10) Art. 56 Rdn. 23 mit dem Hinweis, dass dies auch schon in der Weimarer Republik so vertreten wurde.

47 Maunz/Dürig/Herzog (Fn. 17) Art. 56 Rdn. 13.

48 Maunz/Dürig/Herzog (Fn. 17) Art. 56 Rdn. 13; v. Mangoldt/Klein/ Starck/Fink (Fn. 10) Art. 56 Rdn. 8.

49 Für viele: Schmidt-Bleibtreu/Klein/Butzer (Fn. 10) Art. 56 Rdn. 13; Maunz/Dürig/Herzog (Fn. 17) Art. 56 Rdn. 24.

50 Dolzer/Waldhoff/Graßhof/Fritz (Fn. 15) Art. 56 Rdn. 1; Stern (Fn. 10) $\$ 30$ II $5 \mathrm{a})$.

$51 \mathrm{Vgl}$ nur Art. 42 WRV und $\$ 190$ der Paulskirchenverfassung von 1849.

52 Schmidt-Bleibtreu/Klein/Butzer (Fn. 10) Art. 56 Rdn. 13; Denninger/ Hoffmann-Riem/Schneider/Stein/Jekewitz (Fn. 23) Art. 56 Rdn. 2; v. Mangoldt/Klein/Starck/Fink (Fn. 10) Art. 56 Rdn. 19; Dolzer/Waldhoff/Graßhof/Fritz (Fn. 15) Art. 56 Rdn. 20.

53 Siehe oben C I 1 c).

54 Anders könnte man entscheiden, wenn beispielsweise nur ein Artikel beim Sprechen der Eidesleistung vergessen wird, der Sinn des Eides darunter aber nicht leidet. Im Zweifelsfall sollte aber auch hier der Eid wiederholt werden, um der Verfassung maximal zu entsprechen.

55 Schmidt-Bleibtreu/Klein/Butzer (Fn. 10) Art. 56 Rdn. 23.

56 Denninger/Hoffmann-Riem/Schneider/Stein/Jekewitz (Fn. 23) Art. 56 Rdn. 2.

57 Schmidt-Bleibtreu/Klein/Butzer (Fn. 10) Art. 56 Rdn. 14; v. Mangoldt/ Klein/Starck/Fink (Fn. 10) Art. 56 Rdn. 9.

58 So Dreier/Pernice (Fn. 12) Art. 56 Rdn. 10; v. Münch/Kunig/HemmeRICH (Fn. 23) Art. 56 Rdn. 9; Dolzer/Waldhoff/Graßhof/Fritz (Fn. 15) Art. 56 Rdn. 25ff; Denninger/Hoffmann-Riem/Schneider/Stein/JEKEwitz (Fn. 23) Art. 56 Rdn. 2; Schmidt-Bleibtreu/Klein/Butzer (Fn. 10) Art. 56 Rdn. 14 m. w. N.

59 v. Münch/Kunig/Hemmerich (Fn. 23) Art. 56 Rdn. 9; Schmidt-Bleibtreu/Klein/Butzer (Fn. 10) Art. 56 Rdn. 14 m.w. N. 
gründen verweigert werden $k a_{n}{ }^{60}$. Den entscheidenden Unterschied des Eides des Präsidenten (und des Bundeskanzlers und der Bundesminister) zum gerichtlichen Zeugeneid sieht das BVerfG darin, dass »diese Verpflichtung ... aus dem freiwillig gefassten Entschluss [erwachse], die Wahl in das Amt eines Verfassungsorgans anzunehmen, in dem der Staat in besonderer Weise zu repräsentieren ist und das deshalb grundsätzlich die vollkommene Identifizierung des Gewählten mit den in der Verfassung niedergelegten Wertungen voraussetzt. $\ll^{61}$

Ferner ist der Zugang zu öffentlichen Ämtern in der Spezialnorm Art. 33 III GG geregelt worden, so dass die Glaubensfreiheit aus Art. 4 GG nicht gegen die Eidespflicht aus Art. 56 GG (Art. 64 II GG) in Stellung gebracht werden kann ${ }^{62}$.

Als Ergebnis ist demnach festzuhalten: Der Bundespräsident hat sein Amt zwar wirksam angetreten, dabei jedoch die Verfassung verletzt. Vorgenommene Amtshandlungen sind zwar wirksam, jedoch kann er mit der Präsidentenanklage und der Organklage angeklagt werden.

c) Fall 7: Nach Wahl und Annahme spricht der Bundespräsident vor den versammelten Mitgliedern des Bundestages und Bundesrates den Eid, fügt aber am Schluss der Eidesformel »bei Gott dem Allmächtigen und Allwissenden « noch hinzu.

In diesem Fall hat ein frisch gewählter Bundespräsident vielleicht aus Übereifer, vielleicht aus besonderer Frömmigkeit - dem Eid einen Zusatz hinzugefügt. Auf den ersten Blick erscheint hier die Lage nach dem bisher Ausgeführtem klar: Auch der Zusatz stellt eine vom Grundgesetz nicht vorgesehene Abweichung von der Eidesformel dar. Als solche müsste sie der Eidesverweigerung gleichgestellt werden mit allen bereits erwähnten Folgen. Diese Sichtweise wird auch von weiten Teilen der Literatur so vertreten ${ }^{63}$.

Indes gibt es auch Gegenstimmen, die Zusätze, die dazu dienen, die im Eid übernommenen Verpflichtungen noch zu verstärken, für zulässig erachten ${ }^{64}$. Danach wäre der Eid nur dann unvollständig geleistet, wenn der Bundespräsident solche Zusätze einfügt, die entweder den Sinn und Zweck des Eides zuwider laufen oder durch die er zu erkennen gibt, dass er den Eid, so wie er vorgeschrieben ist, nicht leisten will ${ }^{65}$.

Gegen diese Ansicht lässt sich der klare Wortlaut des Art. 56 GG vorbringen. Andererseits spricht vom Sinn und Zweck des Eides nichts dagegen, wenn der Amtsinhaber durch den Eid seine Verpflichtung aus eigenem Antrieb erhöht. Für die durch Eid angestrebte ethisch-moralische Selbstbindung zugunsten des Staatswohls kann es nur dienlich sein, wenn der Amtsträger seine psychologische Hemmschwelle verstärkt.

Jedenfalls muss es unschädlich sein, wenn der Bundespräsident in der nicht obligatorischen religiösen Beteuerung einen Bezug zu »Gott dem Allmächtigen und Allwissenden « herstellt. Dieser altherkömmliche Zusatz ist beispielsweise auch in Art. 100 Rh.-PfV vorgesehen ${ }^{66}$.

Hierfür spricht auch ein Erst-Recht-Schluss: Wenn die religiöse Beteuerung ganz weggelassen werden kann, so muss erst recht auch ein verstärkender Zusatz erlaubt sein, der zudem auch in einer Landesverfassung vorgesehen ist.

Für Fall 7 bedeutet dies, dass der Bundespräsident den Amtseid korrekt geleistet hat.

\section{Vereidigung des Bundeskanzlers bzw. der Bundes- minister}

\section{Eidesverweigerung}

a) Fall 8: Nach der Wahl durch den Bundestag, erhält der Bundeskanzler vom Bundespräsidenten die Ernennungsurkunde. Die Eidesleistung verweigert der Bundeskanzler nun beharrlich.

Fall 9: Nachdem der Bundeskanzler vom Bundestag gewählt wurde und sowohl Ernennungsurkunde erhalten als auch den Eid geleistet hat, schlägt der Bundeskanzler einen Bundesminister vor. Dieser erhält vom Bundespräsidenten ebenfalls die Ernennungsurkunde. Die Eidesleistung verweigert der Bundesminister aber beharrlich.

In diesen Fällen stellt sich die Frage, ob der Bundeskanzler (Fall 8) bzw. der Bundesminister (Fall 9) sein Amt wirksam angetreten hat.

Das Grundgesetz sagt hierzu in Art. 64 II GG nur aus, dass der Bundeskanzler und die Bundesminister bei der Amtsübernahme vor dem Bundestage den in Art. 56 vorgesehenen Eid zu leisten haben. Damit ist diese Vorschrift fast identisch mit Art. 56 GG, mit der einzigen Ausnahme, dass dort der Eid »bei Amtsantritt « und in Art. 64 II GG »bei Amtsübernahme« zu leisten ist. Nach dem grammatikalischen Wortsinn dürfte aber in beiden Fällen das gleiche gemeint sein. Schon nach dem Volksmund ist zwischen Amtsantritt und Amtsübernahme kein Unterschied erkennbar. Dass für ein und dasselbe verschiedene Formulierungen gewählt worden sind, überrascht umso weniger, als Art. 64 II GG an sich bereits nicht ganz in die Systematik des VI. Abschnitts des Grundgesetzes passt. So müsste die Vereidigung des Bundeskanzlers in Art. 63 GG mitgeregelt sein ${ }^{67}$.

Wie schon bei Art. 56 GG ist damit auch Art. 64 II GG nur zu entnehmen, dass der Eid in unmittelbar zeitlich engem Zusammenhang mit der Amtsübernahme stattfinden soll. Folglich sagt diese Norm nur aus, wann der Eid zu leisten ist, nicht aber, dass das Amt vom Eid abhängt. Wann das Amt aber beginnt, ist nicht ausdrücklich im Grundgesetz geregelt. Jedoch herrscht in der Literatur weitgehend Einigkeit, dass das entscheidende Moment für die Begründung des Amtes die Ernennung des Bundeskanzlers bzw. der Bundesminister durch den Bundespräsidenten gemäß Art. 63 II 2 GG, Art. 64 I GG ist ${ }^{68}$. Diese Ernennung wird dabei durch die Aushändigung der Ernennungsurkunde durch den Bundespräsidenten vollzogen ( $\$ 2$ II Var. 1 BMinG). Die Entgegennahme der Ernennungsurkunde bewirkt also die Amtsübernahme in dem Sinne, dass das öffentlich-rechtliche Amtsverhältnis beginnt ${ }^{69}$. Folglich ist nur die Ernennung, nicht aber der Eid konstitutiv für das $\mathrm{Amt}^{70}$. Die einfachgesetzliche Regelung des $\$ 2$ II Var. 1 BMinG entspricht dem.

Eine Stütze findet diese Ansicht auch im Grundgesetz: So ist sowohl beim Bundeskanzler (Art. 63 II 2 GG) als auch bei den Bundesministern (Art. 64 I GG) die Ernennung systematisch vor der Eidesverpflichtung (Art.64 II GG) genannt, was deutlich macht, dass die Verfassung die Ernennung als konstitutives Ele-

60 BVerfGE 33, $23(28 \mathrm{ff} / 31 \mathrm{f})$.

61 BVerfGE 33, 23, 31

62 Isensee/Kirchhof/Nettesheim (Fn. 16) $\$ 61$ Rdn. 55; ebenfalls in diese Richtung STERN (Fn. 10) \$30 II 5 a).

63 v. Münch/Kunig/Hemmrich (Fn. 23)Art. 56 Rdn. 4; Sachs/Nierhaus (Fn. 10) Art. 56 Rdn. 1; Dreier/Pernice (Fn. 12) Art. 56 Rdn. 10; Maunz/ Dürig/Herzog (Fn. 17) Art. 56 Rdn. 24; Isensee/Kirchhof/Nettesheim (Fn. 16) $\$ 61$ Rdn. 55

64 v. Mangoldt/Klein/Starck/FInK (Fn. 10) Art. 56 Rdn. 19; v. Mangoldt/ KLeIn Das Bonner Grundgesetz, Bd II, 2. Auflage 1966, Art. 56 IV. 2; auch in diese Richtung Dolzer/Waldhoff/Graßhof/Fritz (Fn. 15) Art. 56 Rdn. 20.

65 v. Mangoldt/KLEIN (Fn. 64) Art. 56 IV. 2.

66 Im Gegensatz zum GG lies die WRV dem Reichspräsidenten bei der religiösen Beteuerung einen größeren Spielraum, wenn es dort in Art. 42 WRV hieß: »...Die Beifügung einer religiösen Beteuerung ist zulässig."

67 Maunz/Dürig/Herzog (Fn. 17) Art. 64 Rdn. 28; Dreier/Hermes (Fn. 12) Art. 64 Rdn. 31.

68 Schmidt-Bleibtreu/Klein/Uhle (Fn. 10) Art. 64 Rdn. 18; Sachs/Oldiges (Fn. 10) Art. 64 Rdn. 17; v. Münch/Kunig/Meyn (Fn. 23) Art. 64 Rdn. 15; Dreier/Hermes (Fn. 12) Art. 64 Rdn.26, 31; Friauf/Höfling/Busse (Fn. 38) Art. 63 Rdn. 18, Art. 64 Rdn. 25; F. MÜNCH (Fn. 30) S 168; Isensee/Kirchhof/Schröder (Fn. 16) \$65 Rdn. 22ff, 25.

69 v. Münch/Kunig/Meyn (Fn. 23) Art.64 Rdn. 15; Dreier/Hermes (Fn. 12) Art. 64 Rdn. 26

70 Für viele: Sachs/Oldiges (Fn. 10) Art. 64 Rdn. 17; Schmidt-Bleibtreu/ Klein/Uhle (Fn. 10) Art. 64 Rdn. 18, 29. 
ment betrachtet. Darüber hinaus können die bereits beim Eid des Bundespräsidenten erläuterten Argumente (C I 1 a)) auch hier herangezogen werden. So sprechen der Vergleich mit dem Diensteid des Beamten und die Feststellung, dass aus dem Eid selbst keine weiteren Rechte und Pflichten erwachsen als nach dem Grundgesetz bereits bestehen ${ }^{71}$, für diese Sichtweise.

Soweit $\$ 2$ II Var. 2 BMinG das Amtsverhältnis bereits vor Erhalt der Ernennungsurkunde mit der Eidesleistung beginnen lässt und damit dem Eid konstitutive Wirkung beimisst, entspricht dies nicht der Verfassung und ist daher verfassungswidrig $^{72}$. Zu Recht hat daher die politische Praxis von dieser Regelung bisher keinen Gebrauch gemacht ${ }^{73}$.

Folglich sind sowohl der Bundeskanzler (F. 8) als auch der Bundesminister (F. 9) ohne Eidesleistung wirksam im Amt, sobald sie die Ernennungsurkunde erhalten haben ${ }^{74}$.

b) Freilich stellt die Weigerung, den vorgeschriebenen Eid zu leisten, eine Verletzung der Verfassung dar, die sich auf die von den unvereidigten Amtsträgern vorgenommenen Hoheitsakte auswirkt.

Auch hier herrscht Streit über die Rechtsfolgen hinsichtlich der vorgenommenen Amtshandlungen. Die Diskussion gleicht der, die beim Bundespräsidenten zu dieser Frage geführt wird ${ }^{75}$. Deswegen sei an dieser Stelle nur noch einmal erwähnt, dass insoweit der herrschenden Meinung zu folgen ist, wonach ohne Eid vorgenommene Amtshandlungen zwar unzulässig, aber wirksam $\operatorname{sind}^{76}$.

c) Fall 10: Da sich der Bundeskanzler/Bundesminister vehement weigert, den Amtseid zu leisten, berät man im Bundesrat die möglichen Sanktionen für dieses Verhalten.

\section{aa) Bundeskanzler}

Da das Grundgesetz, anders als ältere deutsche Verfassungen, eine Kanzler- oder Ministeranklage entsprechend der in Art. 61 GG vorgesehenen Präsidentenanklage nicht kennt, ist als erstes an die Organklage durch den Bundestag zu denken (Art. 93 I Nr. 1 GG $)^{77}$. Indes kann in diesem Organstreitverfahren gemäß $\$ 67$ BVerfGG nur die verfassungsrechtliche Pflicht festgestellt werden. Eine Erzwingung der Eidesleistung ist damit nicht mög$\operatorname{lich}^{78}$. Deswegen wird vertreten, dass der Bundespräsident bei einem Verstoß gegen die Eidespflicht berechtigt ist, den Bundeskanzler zu entlassen ${ }^{79}$. Dem Bundespräsidenten ein solches Recht bzw. Pflicht ${ }^{80}$ zuzugestehen, geht jedoch zu weit ${ }^{81}$. Das Grundgesetz erwähnt ein solches Recht oder gar Pflicht nicht. Vielmehr steht es dem Bundestag zu, aus dem Verhalten des Bundeskanzlers entsprechende Konsequenzen zu ziehen mittels eines konstruktiven Misstrauensvotums (Art.67 GG) ${ }^{82}$.

\section{bb) Bundesminister}

Wie schon beim Bundeskanzler besteht auch hier die Möglichkeit, dass der Bundestag gegen den den Eid verweigernden Bundesminister eine Organklage erhebt ${ }^{83}$.

Nach einer Ansicht soll der Bundeskanzler sogar verpflichtet sein, eidesunwillige Minister dem Bundespräsidenten zur Entlassung vorzuschlagen. Denn durch die Eidesverweigerung zeige sich, dass der Minister nicht die nötige Verfassungstreue mit sich bringe. Außerdem würde der Bundeskanzler ansonsten gegen seine eigene Pflicht, für eine verfassungsmäßige Zusammensetzung des Kabinetts Sorge zu tragen, verstoßen ${ }^{84}$.

Jedoch finden diese "Pflichten" des Bundeskanzlers im Grundgesetz keine explizite Erwähnung. Hinzu kommt, dass eine solche »Pflicht « auch mit dem Fehlen von an den Eid geknüpften konstitutiven Rechtswirkungen in gewissem Maße kollidieren würde. Sinnvoller erscheint es deshalb, eine Entlassungspflicht des Kanzlers abzulehnen ${ }^{85}$.
Damit fällt es dem Bundestag zu, dem Verhalten des Bundesministers die entsprechenden politischen Konsequenzen folgen $\mathrm{zu}$ lassen. Jedoch kann der Bundestag nicht direkt gegen den Bundesminister (außer mit der Organklage) vorgehen, sondern nur über den Umweg des konstruktiven Misstrauensvotums gegen den Bundeskanzler (Art.67 I GG). Regelmäßig würde der Bundeskanzler aber, um diesen Fall zu verhindern, dann wohl den eidesverweigernden Bundesminister entlassen ${ }^{86}$.

\section{Abweichung beim Sprechen der Eidesformel:}

Da sich bei der Lösung der drei denkbaren Konstellationen keine Unterschiede ergeben, wird hier auf die entsprechenden Ausführungen beim Bundespräsidenten verwiesen (C I 2 a)-c)).

\section{Ausblick}

Im Amtseid zeigt sich wie in kaum einer anderen Verfassungsbestimmung, welche Politik die Verfassung anstrebt. Die große Bedeutung, welche die US-Verfassung dem Amtseid beimisst, erklärt sich aus der spezifischen gesellschaftlichen Zusammensetzung der USA. Vor dem Hintergrund des »melting pot« legte die US-Verfassung seit jeher besonderen Wert auf Symbolik. Solche symbolischen Feierlichkeiten, wie die Vereidigung der höchsten Staatsorgane, ermöglichen es sowohl den Einwanderern als auch den Einheimischen, sich mit dem Staat zu identifizieren. Mit dem gesellschaftlichen Wandel wird auch hierzulande eine integrative Symbolik immer wichtiger und der Amtseid des Grundgesetzes mehr an Gewicht und Aufmerksamkeit gewinnen.

71 Siehe hierzu Sachs/Oldiges (Fn. 10) Art. 64 Rdn. 17; Schmidt-Bleibtreu/ Klein/Uhle (Fn. 10) Art. 64 Rdn.28; Friauf/Höfling/Busse (Fn. 38), Art. 64 Rdn. 25; Maunz/Dürig/Herzog (Fn. 17) Art. 64 Rdn. 30.

72 v. Mangoldt/Klein/Starck/Schröder (Fn. 10) Art. 63 Rdn. 43, Art. 64 Rdn. 39; Sachs/Oldiges (Fn. 10) Art. 64 Rdn. 17; Schmidt-Bleibtreu/ Klein/Uhle (Fn. 10) Art. 64 Rdn. 18, 29; v. Münch/Kunig/Meyn (Fn. 23) Art. 64 Rdn. 9, 15; Friauf/Höfling/Busse (Fn. 38), Art. 63 Rdn. 18 und Art. 64 Rdn. 25; Dreier/Hermes (Fn. 12) Art. 64 Rdn. 26; Bedenken gegen die Norm bei Dolzer/Waldhoff/Graßhof/Schenke (Fn. 15) Art. 64 Rdn.68; wohl auch Denninger/Hoffmann-Riem/Stein/ScHNeIDER (Fn. 23) Art. 64 Rdn. 7

73 v. Münch/Kunig/Meyn (Fn. 23) Art. 64 Rdn. 15; v. Mangoldt/Klein/ Starck/Schröder (Fn. 10) Art.64 Rdn. 39; Friauf/Höfling/Busse (Fn. 38), Art. 64 Rdn. 25.

74 Dreier/Hermes (Fn. 12) Art. 64 Rdn. 31.

75 Siehe dazu C I 1 c).

76 Für viele: Maunz/Dürig/Herzog (Fn. 17) Art. 64 Rdn. 37; Sachs/OldiGes (Fn. 10) Art. 64 Rdn. 17

77 Maunz/Dürig/Herzog (Fn. 17) Art. 64 Rdn. 29; Sachs/Oldiges (Fn. 10) Art. 63 Rdn. 24.

78 v. Münch/Kunig/Meyn (Fn. 23) Art. 64 Rdn. 16.

79 Dolzer/Waldhoff/Graßhof/Schenke (Fn. 15) Art. 64 Rdn.69; Denninger/Hoffmann-Riem/Stein/Schneider (Fn. 23) Art. 64 Rdn. 7.

80 So Denninger/Hoffmann-Riem/Stein/Schneider (Fn. 23) Art.64 Rdn. 7.

81 Für viele: Sachs/Oldiges (Fn. 10) Art.63 Rdn. 24; v. Münch/Kunig/ Meyn (Fn. 23) Art. 64 Rdn. 16

82 Maunz/Dürig/Herzog (Fn. 17) Art. 64 Rdn. 29; Sachs/Oldiges (Fn. 10) Art. 63 Rdn. 24; v. Münch/Kunig/Meyn (Fn. 23) Art. 64 Rdn. 16; Dreier/ Hermes (Fn. 12) Art. 64 Rdn. 32; Isensee/Kirchhof/Schröder (Fn. 16) $\$ 65 \mathrm{Rdn} .25$.

83 Dreier/Hermes (Fn. 12) Art. 64 Fn. 133.

84 Für viele: Schmidt-Bleibtreu/Klein/Uhle (Fn. 10) Art. 64 Rdn. 30; Sachs/ Oldiges (Fn. 10) Art. 64 Rdn. 17; Denninger/Hoffmann-Riem/Stein/ Schneider (Fn. 23) Art. 64 Rdn. 7.

85 So auch Dreier/Hermes (Fn. 12) Art. 64 Rdn. 32; Maunz/Dürig/Herzog (Fn. 17) Art. 64 Rdn. 29; v. Mangoldt/Klein/Starck/SCHröDer (Fn. 10) Art. 64 Rdn. 40; Friauf/Höfling/Busse (Fn. 38), Art. 64 Rdn. 26. 86 v. Mangoldt/Klein/Starck/SchröDer (Fn. 10) Art. 64 Rdn. 40; Friauf/ Höfling/Busse (Fn. 38), Art. 64 Rdn. 26. 